\title{
Consumo de bebida alcoólica, qualidade de vida e nível de atividade física entre universitários do curso de Educação Física
}

\author{
Alcoholic beverage consumption, quality of life and physical activity level in university students in \\ the Physical Education course \\ Consumo de bebidas alcohólicas, calidad de vida y nivel de actividad física en estudiantes \\ universitarios del curso de Educación Física
}

Recebido: 24/01/2022 | Revisado: 01/02/2022 | Aceito: 10/02/2022 | Publicado: 15/02/2022

João Antônio Ribeiro

ORCID: https://orcid.org/0000-0001-6182-0158 Centro Universitário Governador Ozanam Coelho, Brasil

E-mail: joaoaribeiro1@gmail.com

Denise Coutinho de Miranda

ORCID: https://orcid.org/0000-0001-8714-6309

Universidade Federal de Ouro Preto, Brasil

E-mail: denisecmiranda@gmail.com

Luciano Bernardes Leite

ORCID: https://orcid.org/0000-0002-0885-5834 Universidade Federal de Viçosa, Brasil

E-mail: luciano.leite@ufv.br

Mauro César Isoldi

ORCID: https://orcid.org/0000-0003-4961-5332

Universidade Federal de Ouro Preto, Brasil

E-mail: mauroisoldi@hotmail.com

Thalita Azevedo Cabral

ORCID: https://orcid.org/0000-0001-6265-0840

Centro Universitário Governador Ozanam Coelho, Brasil

E-mail: coordnutri@unifagoc.edu.br

Renata Aparecida Rodrigues de Oliveira

ORCID: https://orcid.org/0000-0002-5004-5253

Centro Universitário Governador Ozanam Coelho, Brasil

E-mail: renata.oliveira@unifagoc.edu.br

Victor Neiva Lavorato

ORCID: https://orcid.org/0000-0001-9914-4722

Centro Universitário Governador Ozanam Coelho, Brasil

E-mail: victor.lavorato@unifagoc.edu.br

\begin{abstract}
Resumo
O consumo de bebida alcoólica é um costume antigo e está presente na vida de parte dos jovens brasileiros de hoje. Nos dias atuais, o álcool tem sido precursor de diversas doenças no planeta. Nesse sentido, o objetivo do presente estudo foi verificar o consumo de bebidas alcoólicas, bem como sua associação com o nível de atividade física e a qualidade de vida em estudantes do curso de Educação Física. Foi realizado um estudo descritivo com 26 universitários regularmente matriculados no curso de Educação Física, sendo aplicados três questionários: um que analisa a percepção subjetiva da qualidade de vida (WHOQOL); outro para avaliar padrões de consumo de bebida alcóolica (AUDIT), e, por fim, um que avalia o nível de atividade física (IPAQ). Pós coleta dos dados feita pelo Google Forms ${ }^{\circledR}$, esses foram analisados. Os resultados mostram que a maioria dos estudantes foram classificados na zona de "Baixo risco" (80,77\%), e que 87,5\% desses são considerados fisicamente ativos. Além disso, não foram encontradas diferenças entre os domínios da qualidade de vida para os estudantes dos grupos classificados como "Baixo risco" ou "Uso de risco". Conclui-se que a maior parte dos avaliados foi classificada como ativa e Baixo risco de consumo de bebidas alcoólicas. O consumo de bebidas alcoólicas não influenciou a qualidade de vida da amostra analisada.
\end{abstract}

Palavras-chave: Atividade física; Atenção; Adolescentes; Ensino.

\begin{abstract}
The consumption of alcoholic beverages is an old custom and is present in the lives of young Brazilians today. Nowadays, alcohol has been a precursor of several diseases on the planet. In this sense, the objective of the present study was to verify the consumption of alcoholic beverages, as well as its association with the level of physical
\end{abstract}


activity and the quality of life in students of the Physical Education course. A descriptive study was carried out with 26 university students regularly enrolled in the Physical Education course, with three questionnaires being applied: one that analyzes the subjective perception of quality of life (WHOQOL); another to assess alcohol consumption patterns (AUDIT), and, finally, one that assesses the level of physical activity (IPAQ). After data collection made by Google Forms ${ }^{\circledR}$, these were analyzed. The results show that most students were classified in the "Low risk" zone $(80.77 \%)$, and that $87.5 \%$ of them are considered physically active. In addition, no differences were found between the quality of life domains for students in the groups classified as "Low risk" or "Use of risk". It is concluded that most of those evaluated were classified as active and Low risk of alcohol consumption. The consumption of alcoholic beverages did not influence the quality of life of the analyzed sample.

Keywords: Physical activity; Heads up; Teenagers; Teaching.

\section{Resumen}

El consumo de bebidas alcohólicas es una costumbre antigua y está presente en la vida de los jóvenes brasileños de hoy. En la actualidad, el alcohol ha sido precursor de varias enfermedades en el planeta. En ese sentido, el objetivo del presente estudio fue verificar el consumo de bebidas alcohólicas, así como su asociación con el nivel de actividad física y la calidad de vida en estudiantes del curso de Educación Física. Se realizó un estudio descriptivo con 26 estudiantes universitarios matriculados regularmente en la carrera de Educación Física, aplicándose tres cuestionarios: uno que analiza la percepción subjetiva de la calidad de vida (WHOQOL); otro para evaluar patrones de consumo de alcohol (AUDIT), y, finalmente, uno que evalúa el nivel de actividad física (IPAQ). Luego de la recolección de datos realizada por Google Forms ${ }^{\circledR}$, estos fueron analizados. Los resultados muestran que la mayoría de los estudiantes fueron clasificados en la zona de "Bajo riesgo" (80,77\%), y que el 87,5\% de ellos se consideran físicamente activos. Además, no se encontraron diferencias entre los dominios de calidad de vida de los estudiantes en los grupos clasificados como "Bajo riesgo" o "Uso de riesgo". Se concluye que la mayoría de los evaluados fueron clasificados como activos y de bajo riesgo de consumo de alcohol. El consumo de bebidas alcohólicas no influyó en la calidad de vida de la muestra analizada.

Palabras clave: Actividad física; Aviso; Adolescentes; Enseñando.

\section{Introdução}

O álcool é uma das substâncias psicoativas mais consumidas no mundo, principalmente entres os jovens universitários (Word Health Organization, 2014). Historicamente, segundo o Centro Brasileiro de Informações sobre Drogas Psicotrópicas (CEBRID, 2010) o consumo de álcool pelo ser humano é um costume antigo e persistente por milhares de anos. Registros arqueológicos revelam indícios de seu consumo aproximadamente 6000 a.C., inicialmente com bebidas de baixo teor alcoólico, como, por exemplo, a cerveja e o vinho (CEBRID, 2010).

$\mathrm{Na}$ atualidade o uso de álcool é um dos principais precursores de doenças no planeta. Cerca de $10 \%$ de mortes entre pessoas de 15 a 49 anos são advindas de seu uso. Desse modo, quando se trata de estudantes nessa faixa etária, eles alegam que o uso dessa droga está ligado à fuga de problemas cotidianos, a situações negativas de cunho pessoal, à pressão social de forma direta e indireta e, principalmente, ao prazer que a bebida traz ou da situação em que ela é consumida (Barros et al., 2012; Griswold et al., 2016). No Brasil estudos epidemiológicos mostraram elevadas prevalências de consumo de álcool, que variam de 66,3\% a 91,9\% (Kerr-Corrêa et al., 1999; Pedrosa et al., 2011).

O álcool, em tese, é a droga preferida entre os jovens, e esses, teoricamente, estão iniciando seu consumo cada vez mais cedo. Com a inserção desses jovens nas universidades, muitos passam a ter a primeira oportunidade de viver suas vidas longe de seus familiares. Com a alta entrada de jovens em universidades, ocorrem diversas mudanças em suas vidas, principalmente para os que se afastam de casa. Novos hábitos são adquiridos e alteram seu estilo de vida por tempo suficiente para trazer danos à saúde. Nas universidades, geralmente se encontram os jovens que tiveram uma adolescência carregada de sentimentos que os deixaram mais vulneráveis ao consumo de álcool (Silva et al., 2006; Wagner et al., 2008; Moraes et al., 2010; Pedrosa et al., 2011; Silva, 2015).

Diante do exposto, estudos que avaliem o consumo de bebidas alcoólicas, a qualidade de vida e o nível de atividade física de estudantes do ensino superior podem ser fundamentais, tendo em vista a cobrança com as aulas e a sobrecarga com o trabalho, fazem com que os estudantes gastem menos tempo cuidando de sua saúde, tornando-se inativos fisicamente e 
adquirindo diversos tipos de doenças. Ademais, tais dados podem trazer um panorama geral da situação dos estudantes para que se crie programas de prevenção ao consumo abusivo de álcool, que se incentive a prática de atividade física e a boa alimentação para a manutenção e melhora da qualidade de vida. Dessa forma o presente estudo objetivou verificar o consumo de bebidas alcoólicas, bem como sua associação com o nível de atividade física e a qualidade de vida em estudantes do curso de Educação Física.

\section{Metodologia}

\section{Amostra}

Foi realizado um estudo descritivo com delineamento transversal realizado com 26 estudantes regularmente matriculados do curso de Educação Física de um Centro Universitário da cidade de Ubá - MG a pelo menos 6 meses, sem critérios de exclusão para etnia, faixa etária e sexo. Foram excluídos do estudo aqueles que não concordaram em participar do estudo ou não responderam a todos os questionários.

\section{Procedimentos}

A coleta de dados foi feita por meio de questionários online através da plataforma Google Forms ${ }^{\circledR}$ devido ao não término da pandemia.

Inicialmente uma carta de solicitação de pesquisa foi enviada ao Centro Universitário para ser dada a autorização devida para a realização da pesquisa. Para a confirmação do interesse em participar da pesquisa, foi aplicado o Termo de Consentimento Livre Esclarecido, obedecendo às normas para a realização de pesquisas em seres humanos, resolução ${ }^{\circ}$ 466/12 do Conselho Nacional de Saúde.

\section{Instrumentos}

\section{Qualidade de vida}

Para a avaliação da Qualidade de Vida (QV) dos universitários, utilizou-se o Instrumento de Avaliação de Qualidade de Vida da Organização Mundial da Saúde (WHOQOL - BREF), traduzido e validado para o português por Fleck et al., (2000). O questionário é composto por 4 domínios: físico, psicológico, relações sociais e ambiente; é realizado através de 26 questões, onde a primeira questão refere-se à QV de modo geral e a segunda questão refere-se à satisfação com a própria saúde e as 24 questões restantes representam uma das 24 facetas do instrumento original. As respostas seguem uma escala de Likert (de 1 a 5, quanto maior a pontuação, melhor a qualidade de vida). Em cada faceta foi somado os valores da entrevista e dividido pelo número de participantes onde foi realizado uma média em que o resultado foi de 1 a 5 . A classificação foi dada através dos valores: Necessita melhorar (1 a 2,9); Regular (3 a 3,9); Boa (4 a 4,9); Muito boa (5) (Fleck et al., 2000). Posteriormente, esses valores foram transformados em porcentagem simples.

\section{Consumo de bebida alcóolica}

Para avaliar padrões de consumo de bebida alcóolica foi utilizado o Alcohol Use Disorders Identification Test (AUDIT), adaptado para o Brasil por Méndez (1999). O AUDIT é composto por 10 itens de múltipla escolha que variam de 0 a 4 permitindo, assim, margem de pontuação de 0 a 40. A partir da pontuação do participante, nessa escala de medida, é possível classificá-lo em quatro zonas (padrões) de consumo: baixo risco - 0 a 7 pontos; uso de risco - 8 a 15 pontos; uso nocivo - 16 a 19; e dependência - 20 a 40 pontos (Babor et al., 2001). 


\section{Nível de atividade física}

Para avaliar o nível de atividade física utilizou-se o Questionário Internacional de Atividade Física (IPAQ - versão curta). Em relação ao IPAQ, os universitários foram classificados em fisicamente ativos e insuficientemente ativos. O questionário é constituído por 4 perguntas, sendo cada uma composta por letras A e B. As perguntas estão relacionadas às atividades realizadas na semana anterior à aplicação do teste (Matsudo et al., 2001). De acordo com o IPAQ, o indivíduo pode ser classificado como muito ativo, ativo, irregularmente ativo A, irregularmente ativo B e sedentário.

\section{Nível de atividade física}

Os dados foram apresentados como média e desvio padrão da média e porcentagem simples. Os grupos de estudantes foram divididos em "Ativos" (ativos + muito ativos) e "Não ativos" (sedentários + irregularmente ativos A e B). Inicialmente realizou-se um teste para verificação da normalidade dos dados. Posteriormente, utilizou-se o teste $T$ de Student para comparação dos dados de qualidade de vida. O nível de significância adotado foi de 5\%. Os dados foram analisados através do programa estatístico Graph Pad Prism 8.0 ${ }^{\circledR}$.

\section{Resultados}

Foram obtidas 26 respostas de alunos que concordaram em participar da pesquisa. Dentre os homens que participaram, $82,35 \%$ (14) consomem bebida alcoólica, enquanto as mulheres que participaram, 55,55\% (5) consumem de bebida alcoólica.

A Tabela 1 apresenta os dados relativos ao sexo e à idade dos participantes. A maior parte da amostra foi composta por homens e com idade de 18 a 25 anos.

Tabela 1. Caracterização da amostra.

\begin{tabular}{lc}
\hline Sexo & $\%$ \\
\hline Masculino & $65,4 \%$ \\
Feminino & $34,6 \%$ \\
Faixa etária & $\%$ \\
18 a 25 anos & $69,2 \%$ \\
26 a 30 anos & $19,2 \%$ \\
31 a 35 anos & $3,8 \%$ \\
36 a 40 anos & $0 \%$ \\
41 a 45 anos & $3,8 \%$ \\
46 a 50 anos & $3,8 \%$ \\
\hline
\end{tabular}

Fonte: Dados da pesquisa (2021).

A Tabela 2 apresenta os dados do questionário AUDIT. O questionário aplicado utiliza um sistema de pontos atribuídos a cada resposta, desse modo, os alunos são classificados quanto ao nível de consumo de álcool durante os últimos 12 meses. De acordo com os dados obtidos, a maior parte da amostra (80,77\%) foi classificado como "Baixo risco". 
Tabela 2. Classificação do questionário AUDIT.

\begin{tabular}{lc}
\hline Classificação & Inativo (\%) \\
\hline Baixo risco & 80,77 \\
Uso de risco & 19,23 \\
Uso nocivo & $0 \%$ \\
Dependência & $0 \%$ \\
\hline
\end{tabular}

Fonte: Dados da pesquisa (2021).

A Tabela 3 apresenta a relação entre o AUDIT e classificação do nível de atividade física do IPAQ divididos em Inativo e Ativo. Dentre os estudantes classificados como "Baixo risco" no AUDIT, a maior parte foi classificada como ativa no IPAQ. Por outro lado, aqueles classificados como "Uso de risco" no AUDIT, a maior parte foi classificada como inativa no IPAQ (Tabela 3).

Tabela 3. Relação entre o AUDIT e a classificação do nível de atividade física classificados como Inativo e Ativo.

\begin{tabular}{lcc}
\hline Classificação & Inativo (\%) & Ativo (\%) \\
\hline Baixo risco & $12.5 \%$ & $87,5 \%$ \\
Uso de risco & $60,0 \%$ & $40,0 \%$ \\
Uso nocivo & $0 \%$ & $0 \%$ \\
Dependência & $0 \%$ & $0 \%$ \\
\hline
\end{tabular}

Fonte: Dados da pesquisa (2021).

A Tabela 4 indica os domínios da qualidade de vida através do questionário WHOQOL - BREF para os indivíduos classificados como "Baixo risco" e "Uso de risco" no AUDIT. Não houve diferenças significativas entre os dois grupos.

Tabela 4. Domínios da Qualidade de Vida e Qualidade de Vida Global.

\begin{tabular}{lcc}
\hline Dominíos & Baixo risco & Uso de risco \\
\hline Físico & $55,19 \pm 8,79$ & $48,57 \pm 13,02$ \\
Psicológico & $62,69 \pm 9,31$ & $67,50 \pm 15,98$ \\
Relações Sociais & $65,91 \pm 15,98$ & $65,91 \pm 15,62$ \\
Meio Ambiente & $47,30 \pm 4,23$ & $42,50 \pm 16,77$ \\
QV Global & $57,77 \pm 6,26$ & $55,06 \pm 16,38$ \\
\hline
\end{tabular}

Fonte: Dados da pesquisa (2021).

\section{Discussão}

O presente estudo verificou o consumo de bebidas alcoólicas, bem como sua associação com o nível de atividade física e a qualidade de vida em estudantes do curso de Educação Física.

Os dados obtidos nesta pesquisa evidenciam que 73,07\% dos alunos consumiram álcool pelo menos uma vez nos últimos 12 meses. Entre esses universitários, 80,77\% foram classificados como "Baixo risco" no AUDIT não havendo assim, necessidade de intervenção em nenhum dos casos. Esses achados estão em consonância com o estudo de Sousa, Medeiros, Medeiros (2020), que, utilizando diferentes modelos estruturais (uni, bi e tri fatorial), com 406 estudantes universitários, encontraram que $74,4 \%$ da amostra total foi classificada na zona de consumo de álcool de baixo risco. Assim como o estudo feito por Fagundes et. al (2020) com 150 universitários dos cursos de Medicina e Enfermagem, onde foi encontrado que a 
maior parte dos indivíduos da amostra foram classificados como baixo risco. Além disso, os trabalhos de Karno, Granholm e Lin (2000) e Lima et al. (2005), onde utilizaram um modelo de estrutura com dois e três fatores encontrando o resultado de sua amostra, o consumo de baixo risco.

O presente estudo encontrou maior prevalência de consumo de álcool entre homens sendo 82,35\% (14), enquanto o sexo feminino o consumo foi de 55,55\% (5). O resultado é corroborado com um estudo realizado por Carneiro et al. (2014), onde verificou-se que o consumo nocivo e a dependência de álcool é maior entre o sexo masculino em relação ao feminino, o que é confirmado também pelo Ministério da Saúde (2020), ao afirmar que no conjunto das capitais brasileiras a frequência do consumo abusivo de bebidas alcoólicas foi de 18,8\% do total da amostra, sendo maior em homens (25,3\%), pois esses bebem por pressão social, do que em mulheres (13,3\%), pois essas bebem por questões emocionais, e, em ambos os sexos, em que essa frequência diminui com a idade (devido à maior maturidade e diminuição da capacidade fisiológica), e aumenta com o nível de escolaridade (devido ao fato de proporcionar uma melhor renda aos consumidores de bebida) (Brasil, 2020).

Diante do contexto atual, de acordo com a Secretaria de Estado de Desenvolvimento Social de Minas Gerais (SEDESE, 2021), durante a pandemia do COVID-19, o consumo de álcool aumentou em Minas Gerais. Dados divulgados durante audiência pública da Comissão de Prevenção e Combate ao Uso de Crack e Outras Drogas da Assembleia Legislativa de Minas Gerais (ALMG), mostram que a venda de bebidas alcoólicas subiu neste período. Segundo a pasta, o crescimento foi de $38 \%$ nas distribuidoras, $27 \%$ nas lojas de conveniência e $26 \%$ nos serviços de entrega em domicílio, ficando evidente o maior consumo de bebida (UFMG, 2020).

Em seu estudo, Manzatto et al. (2011) afirmam que a Qualidade de Vida (QV) pode ser diferenciada a partir de seus objetivos, formas de abordagem, resultados observados, e as diversas interpretações referentes ao contex to em que é estudada ou aplicada. O estilo e os hábitos de vida, que são formados e adquiridos de acordo com as vivências, ambiente, cultura e costumes em que a pessoa está inserida, podem influenciar de maneira tanto positiva quanto negativa na QV. O consumo abusivo do álcool pode ser encarado como uns dos aspectos que pode comprometer a qualidade de vida dos jovens. A maneira como o álcool influencia a saúde e a QV das pessoas tem despertado interesse, visto que esse é um contexto preocupante principalmente quando analisamos os malefícios causados pelo consumo abusivo do álcool.

Nos tempos atuais, a preocupação sobre um bom estilo de vida para se ter uma boa qualidade de vida está cada vez maior. Nesse sentido, estudos sobre o tema estão cada vez mais sendo realizados. No trabalho de Veiga, Cantorani, Vargas (2016), encontrou-se resultados expressivos ao analisar a qualidade de vida utilizando o WHOQOL-Bref em uma população de acadêmicos de Educação Física. Os autores obtiveram resultados positivos nos domínios físico, psicológico, relações sociais, meio-ambiente e QV em geral. Sendo o resultado mais expressivo o domínio físico onde 76,38\% demonstram que estão satisfeitos com sua condição física, realizando suas tarefas, praticando atividades físicas e tirando um tempo para descanso, o que se confirma nos estudos de Damasceno et al. (2016), ao encontrar que 71,4\% da população de acadêmicos do curso de Educação Física de uma universidade estadual está satisfeita com a realização de suas atividades do dia a dia.

Na presente pesquisa utilizando o WHOQOL-Bref, foram encontrados menores índices no domínio Meio Ambiente para ambos os grupos (Baixo risco e Uso de risco), nos demais domínios foi mantida uma média de aproximadamente $60 \%$. Vale considerar que o resultado do domínio físico foi abaixo do esperado, pois era aguardado um escore maior levando em consideração uma amostra limitada a alunos de educação física, mas isso pode ser explicado pelo fato da amostra estar vivenciando uma pandemia no momento da coleta, o que aumentou o distanciamento social, fechamento de academias e áreas de lazer. Dessa forma, pode ser que os avaliados tenham apresentado maior inatividade física e comportamentos sedentários.

Por fim, ao analisar o nível de atividade física da amostra, revelou-se que dos alunos classificados como "Baixo risco", a maior parte $(87,5 \%)$ foi classificada como ativa (praticante regular de atividade física). Por outro lado, os alunos classificados como "Uso de risco", a maior parte (60\%) foi classificada como inativa (praticante irregular de atividade física). 
Um dado alarmante ao repararmos a diferença que a atividade física faz na vida dos universitários, pois quanto mais inativo ele parece ser mais propenso a fazer uso nocivo de bebida alcóolica.

Uma limitação do estudo foi o baixo número de participantes, o que pode ter reduzido a fidedignidade dos dados, somado à pesquisa online que dificulta a explicação direta do motivo e importância da pesquisa.

\section{Conclusão}

Este estudo teve como objetivo verificar o consumo de bebidas alcoólicas, bem como sua associação com o nível de atividade física e a qualidade de vida em estudantes do curso de Educação Física. Observa-se que a maior parte dos avaliados foi classificada como ativa e no baixo risco do consumo de bebidas alcoólicas. O consumo de bebidas alcoólicas não influenciou a qualidade de vida da amostra analisada.

A partir destes resultados há a necessidade de desenvolvimento de estratégias para incentivar os jovens a reduzirem o consumo de bebidas alcoólicas e aumentar a prática de atividade física. Contudo fazem-se necessários novos estudos com universitários de outros cursos para melhor compreensão da função do exercício sobre o consumo de bebidas alcoólicas.

\section{Referências}

Babor, T. F., Higgins-Biddle, J. C., Saunders, J. B., Monteiro, M. G. (2001). The alcohol use disorders identification test (pp. 1-37). Geneva: World Health Organization.

Barros, C. V. L, Barros, D. A. C., Bernardes, M. J., de Lima, W. V., Silva, L. C. S. (2012). A influência do convívio universitário na adesão ao alcoolismo. Itinerarius Reflectionis, 8(2).

Brasil. Ministério da Saúde. (2020). Estimativas sobre frequência e distribuição sociodemográfica de fatores de risco e proteção para doenças crônicas nas capitais dos 26 estados brasileiros e no Distrito Federal em 2019. https://www.gov.br/saude/pt-br/centrais-de-conteudo/publicacoes/publicacoessvs/vigitel/relatorio-vigitel-2020-original.pdf/view.

Carneiro, A. L. M., Rodrigues, S. B., Gherardi-Donato, E. C. S., de Azevedo Guimarães, E. A., Oliveira, V. C. (2014). Padrão do uso de álcool entre estudantes universitários da área da saúde. Revista de Enfermagem do Centro-Oeste Mineiro.

CEBRID - Centro Brasileiro sobre Drogas Psicotrópicas. (2003). Livreto informativo sobre drogas psicotrópicas. Livreto informativo sobre drogas psicotrópicas. São Paulo, 2003.

Damasceno, R. O., Boery, R. N. S. D., Ribeiro, Í. J. S., Ferraz dosAnjos, K., Santos, V. C., Boery, E. N. (2016). Uso de álcool, tabaco e outras drogas e qualidade de vida de estudantes universitários. Revista Baiana de Enfermagem, 30(3).

Fagundes, L. C., Paz, C. J. R., Freitas, D. A., Barbosa, H. A., Soares, W. D. (2020). Consumo de álcool entre universitários na cidade de Montes Claros-MG. Arquivos Catarinenses de Medicina, 49(3), 12-22.

Fleck, M., Louzada, S., Xavier, M., Chachamovich, E., Vieira, G., Santos, L., Pinzon, V. (2000). Aplicação da versão em português do instrumento abreviado de avaliação da qualidade de vida" WHOQOL-bref". Revista de saúde pública, 34, 178-183.

Griswold, M. G., Fullman, N., Hawley, C., Arian, N., Zimsen, S. R., Tymeson, H. D., Farioli, A. (2018). Alcohol use and burden for 195 countries and territories, 1990-2016: a systematic analysis for the Global Burden of Disease Study 2016. The Lancet, 392(10152), 1015-1035.

Karno, M., Granholm, E., Lin, A. (2000). Factor structure of the Alcohol Use Disorders Identification Test (AUDIT) in a mental health clinic sample. Journal of studies on alcohol, 61(5), 751-758.

Kerr-Corrêa, F., Andrade, A. G. D., Bassit, A. Z., Boccuto, N. M. V. F. (1999). Uso de álcool e drogas por estudantes de medicina da Unesp. Brazilian Journal of Psychiatry, 21, 95-100.

Lima, C. T., Freire, A. C. C., Silva, A. P. B., Teixeira, R. M., Farrell, M., Prince, M. (2005). Concurrent and construct validity of the AUDIT in an urban Brazilian sample. Alcohol and Alcoholism, 40(6), 584-589.

Manzatto, L., Rocha, T. B. X. (2011). Consumo de álcool e qualidade de vida em estudantes universitários. Conexões, 9(1), $37-53$.

Matsudo, S; Araújo, T., Matsudo, V., Andrade, D., Andrade, E., Oliveira, L.C., Braggion, G. (2001). Questionário Internacional de Atividade Física (IPAQ): estudo de validade e reprodutibilidade no Brasil. Revista Brasileira de Atividade Física e Saúde, 6(2), 5-18.

Méndez, E. B. (1999). Uma versão brasileira do AUDIT - Alcohol Use Disorders Identification Test. Dissertação de Mestrado. Universidade Federal de Pelotas, RS.

Moraes, M., de Laat, E. F., de Lara, L., Leite, G. T. (2009). Consumo de álcool, fumo e qualidade de vida: um comparativo entre universitários. Cinergis, $10(1)$. 
Research, Society and Development, v. 11, n. 3, e13011326089, 2022

(CC BY 4.0) | ISSN 2525-3409 | DOI: http://dx.doi.org/10.33448/rsd-v11i3.26089

Pedrosa, A. A. D. S., Camacho, L. A. B., Passos, S. R. L., Oliveira, R. D. V. C. D. (2011). Consumo de álcool entre estudantes universitários. Cadernos de Saúde Pública, 27, 1611-1621.

SEDESE - Secretaria de Estado de Desenvolvimento Social. (2021). Com foco nos adolescentes e jovens, Semana Estadual de Prevenção às Drogas aposta em vida saudável. Belo Horizonte - MG. https://social.mg.gov.br/acessibilidade/story/1117-1501-patrocinio-ganha-novo-posto-do-sine.

Silva, J. N., Rodrigues, M. G., Jones, K. M., Finelli, L. A. C., Soares, W. D. (2016). Consumo álcool entre universitários. Revista Brasileira de Pesquisa em Ciências da Saúde, 2(2), 35-40.

Silva, L.V, Malbergier, A., Stempliuk, V.D.A, Andrade, A.G.D (2006). Fatores associados ao uso de drogas e álcool entre universitários. Revista de saúde pública , 40 (2), 280-288.

Sousa, K. P. A., de Medeiros, E. D., de Medeiros, P. C. B. (2020). Validade e confiabilidade do Alcohol Use Disorders Identification Test (AUDIT) em estudantes de uma universidade brasileira. Ciencias Psicológicas.

UFMG - Universidade Federal de Minas Gerais. (2020). Consumo de álcool aumenta durante isolamento social: vendas em Minas Gerais cresceram até 38\%. https://medicina.ufmg.br.

Veiga, C., Cantorani, J. R. H., Vargas, L. M. (2016). Qualidade de vida e alcoolismo: um estudo em acadêmicos de licenciatura em educação física. Conexões, 14(1), 20-34.

Wagner, G. A., Andrade, A. G. D. (2008). Uso de álcool, tabaco e outras drogas entre estudantes universitários brasileiros. Archives of Clinical Psychiatry (São Paulo), 35, 48-54.

Word Health Organization: Global Status Report on Alcohol and

http://apps.who.int/iris/bitstream/10665/112736/1/9789240692763_eng.pdf?ua=1 . 